\title{
Research on Integrated Energy Simulation System of Distribution Network
}

\author{
Peng Yu ${ }^{1, *}$, Yuejiao Wang ${ }^{1}$, Hao Sun ${ }^{2}$, Mengyang $\mathrm{Li}^{2}$, Rong $\mathrm{Li}^{3}$, and Shumin Sun ${ }^{1}$ \\ ${ }^{1}$ State Grid Shandong Electric Power Research Institute, China \\ ${ }^{2}$ NARI Group Corporations, NARI Research Institute, China \\ ${ }^{3}$ State Grid Shandong Electric Power Company, China
}

\begin{abstract}
This article discussed multiple energy flow simulation technology based on the research status of integrated community energy system. Under the unified platform architecture, simulation, planning, optimization, and analysis of distribution multiple energy flow are realized. According to the idea of three cascades of energy utilization, the modeling of equipment in various professional fields is completed under unified software architecture, and the simulation system is finally developed. The system is built with plugin software, which is open and extensible. The simulation system developed in this paper can be used in the simulation of distribution integrated energy system to realize the interaction between energy flow and information flow.
\end{abstract}

\section{Introduction}

At present, the driving force of economic development is shifting from traditional industries to new fields represented by the Internet and clean energy. Efficient use of energy is the foundation of economic development. Therefore, a boom in the construction of an Energy Internet is emerging worldwide. Energy Internet can achieve green and low-carbon development, which is the correct way to achieve efficient use of energy. During the construction of the Energy Internet, the integrated community energy system (ICES) of distribution has become a focus of attention. ICES, which can make full use of the natural resources on the user side, is formed by coupling energy supply networks such as medium and low voltage distribution systems, medium and low voltage natural gas systems, heat-cold systems. Joint operation of ICES can improve energy efficiency, which is important for the construction of Energy Internet and environment-friendly society. ${ }^{[1-4]}$

Integrated energy modeling and simulation is the basis for ICES planning and operation control. The ICES involves multiple energy sources, so the system has various forms and characteristics. It contains both easyto-control energy and difficult-to-control energy. It contains not only energy that is difficult to store in large capacity, but also energy that is easy to transfer and store. It includes not only the dynamic processes of equipment and systems, but also the more complex dynamic processes of regional comprehensive energy systems. Therefore, the modeling and simulation of ICES are extremely complex. ${ }^{[5-9]}$
At present, there is some commercial software that can be used for ICES. On the basis of electric power simulation, these software have expanded other fields involved in integrated energy, and have some basic functions of integrated energy simulation. However, these software can't well realize the electric-heat-coldgas joint simulation, and can't meet the research needs of more complicated control strategies and optimized operation. Among them, the commercialized software includes: NEPLAN, HOMER, ENERGYPLUS, TRNSYS and PSS®SINCAL.In actual work such as planning, design, and energy-saving analysis, the independent analysis method of each professional software is still used.

Aiming at the current status of the technology, this article has developed an ICES simulation system, which provides auxiliary tools for modeling, simulation, planning, and optimization analysis. With the deepening of research, new forms of energy may appear in the future. The software system needs to have a good openness to facilitate subsequent expansion of the application field.

\section{Demand analysis}

According to the investigation of simulation optimization software in the energy field at home and abroad, combined with the actual development needs of the future integrated energy system, this paper determines functional requirements of integrated energy simulation system:

\footnotetext{
* Corresponding author: 167274738@qq.com
} 
(1) It needs to establish data-driven source and load prediction models to provide accurate power generation and load data for planning, simulation and operation;

(2) It needs to establish an operation simulation platform that integrates power simulation, heat-cold simulation, gas simulation, and joint simulation. At the same time, based on the simulation platform, it can provide functions such as planning and decision-making, operation optimization, and safety analysis;

(3) It needs to establish a unified model system and a complete comprehensive energy system component model library to provide support for basic analysis and advanced applications;

(4) It needs to establish an operation optimization simulation model based on multiple objective and multiple network security constraints, to realize the optimal allocation of integrated energy resources, and to effectively guarantee the safe and stable, economic and green operation of the integrated energy network;

(5) It needs to establish a comprehensive energy equipment-level safety analysis and fault warning system, to provide health management means for the safe and stable operation of the integrated energy system.

To sum up, in the development process of integrated energy simulation system, unified platform modeling is the core of the simulation system, and the effective multiple energy flow interaction mechanism is the key technology for system implementation.

In order to provide users with a good environment for use, this software system adopts a unified style user graphical interface. The system has graphic model establishment, modification, conversion and other model management functions. It can also flexibly and conveniently set simulation parameters and processes. It also has the functions of visualizing simulation results and converting output file formats.

The integrated energy system involves many types of energy. With the deepening of research, new forms of energy may appear in the future. The software system needs to have a good openness to facilitate subsequent expansion of the application field. The software system developed in this paper can call third-party software, enrich the system functions, and realize joint simulation in the fields of electricity, heat, cold and gas.

This paper studies and implements simulation analysis across multiple physical fields, and uses effective methods to integrate different types of energy systems, which can correctly express the operating mechanism and characteristics of integrated energy systems.

\section{Joint simulation}

In order to realize the electricity-heat-cold-gas joint simulation, this paper develops a simulation scheduling manager based on FMI (Functional Mock-up Interface) technology. The simulation scheduling manager mainly includes two functions: simulation step adjustment and coupling equipment data interaction. The step size adjustment realizes the coordination of simulation step sizes between different functional FMUs (Functional Mock-up Units). The data exchange function can exchange real-time simulation data in different function FMUs, which can ensure orderly update of data and improve the accuracy of joint simulation. In the joint simulation process, the simulation operation data of the power FMU, gas FMU and heat-cold FMU can be output through the reserved data access interface to realize the transfer between the modules.

As is shown in Figure 1, the functions of the joint simulation system include: power simulation, gas simulation and heat-cold simulation. The system can not only perform power, heat-cold, and gas simulation alone, but also package the FMU according to the needs of joint simulation to achieve joint simulation.

The FMI standard package retains the professionalism and integrity of system simulation tools in various professional fields, provides a standard calling interface for the simulation scheduling manager to enhance the coupling characteristics of the system.

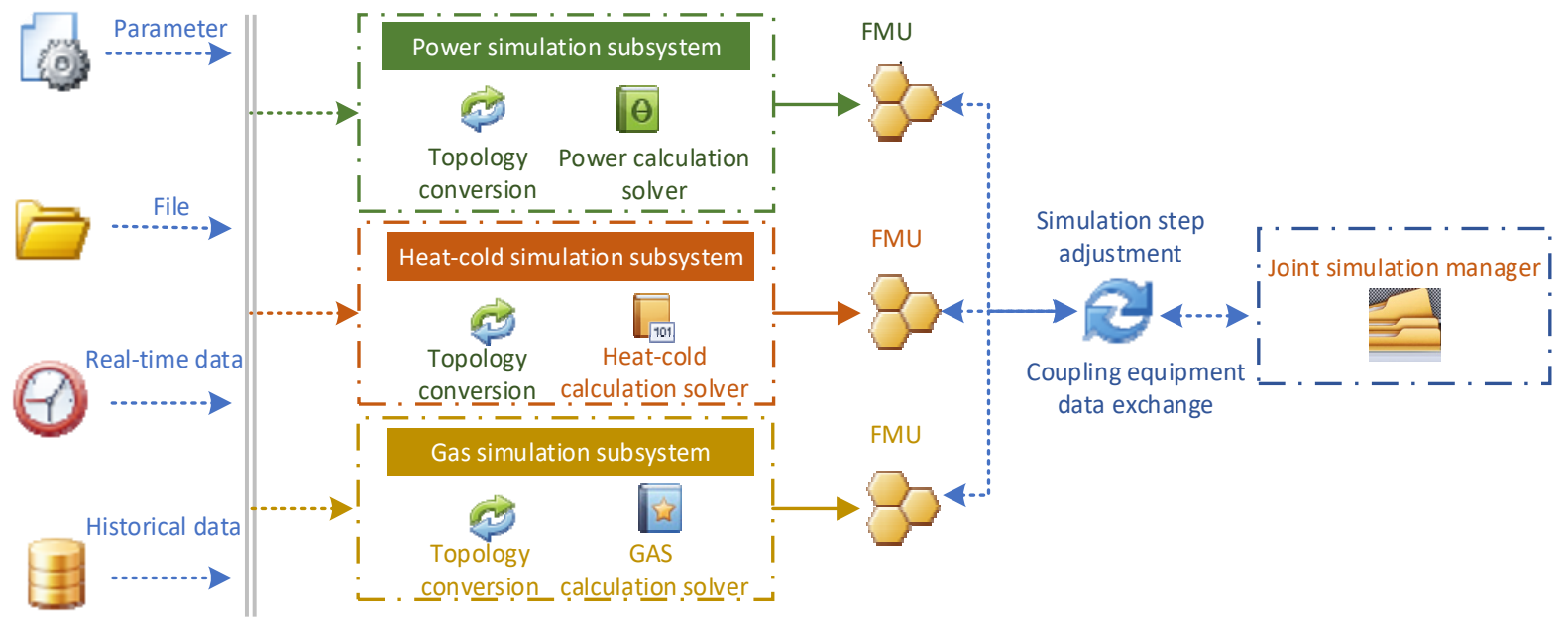

Fig.1. Functions of the joint simulation. 


\section{Energy cascade utilization}

According to the principle of energy flow and cascade utilization, an integrated energy simulation system model is established, as is shown in figure 2 below. This article divides energy utilization into three gradients: primary energy, secondary energy and terminal energy. The primary energy sources mainly include: coal, oil, natural gas, water energy, solar energy, wind energy, geothermal energy, ocean energy, biomass energy, etc.; secondary energy sources are: electricity and hydrogen; terminal energy sources are: cold and heat.

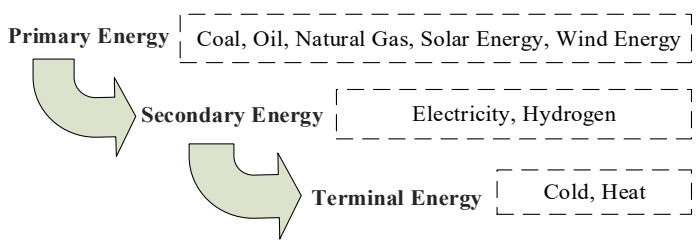

Fig.2. Energy flow and cascade utilization.

According to the principle of one-way flow of energy from low level to high level, design of ICES simulation system can be carried out in steps. At the same time, according to the rules of different cascade energy flows, the energy conversion system and equipment in ICES are coded. Energy flow rules define the inputs and outputs of various types of systems or equipment, thus simplifying complex integrated energy subsystems. It is beneficial to the program design and development.

\section{Simulation analysis process}

(1) Based on the idea of energy cascade utilization, multiple energy flow equipment is selected to establish multiple energy flow network coupling nodes;

(2) In different energy networks, establish their own network and system models;

(3) FMU is formed based on the standard packaging method of FMI;

(4) Through the simulation manager, the simulation step size is set adaptively;

(5) The simulation scheduling manager implements the scheduling management strategy and coordinates the joint simulation of multiple FMUs.

\section{System structure}

The software system constructed in this paper has the characteristics of application-oriented, safe and reliable, flexible expansion, high openness and easy maintenance. The plug-in architecture of the software system is shown in Figure 4.

This software system is built using plug-in-based software and is a multiple level plug-in framework. The first-level plug-in is connected to the plug-in manager; the second-level plug-in is connected to the corresponding first-level plug-in, and so on.

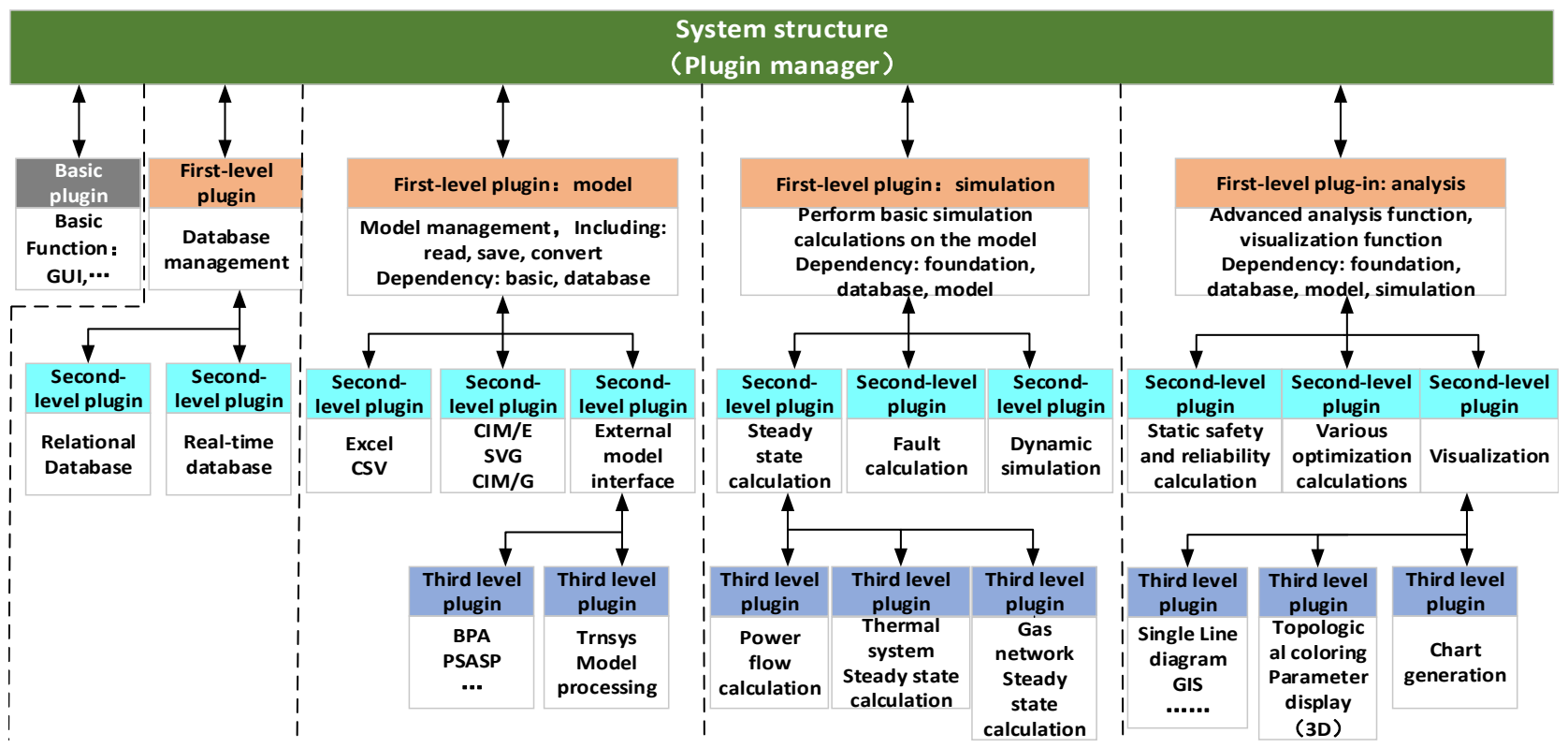

Fig.3. Plug in architecture of software system.

The plug-in manager is responsible for the loading, destruction and management of each plug-in. But the plug-in manager does not involve any specific system functions, such as models, simulation algorithms, data visualization, etc. This software system needs to complete the development of the plug-in manager first, and subsequent business functions based on different plug-ins may work properly.

The core plug-in is responsible for implementing the basic functions of the software system and the plug-in architecture, and providing support for the plug-ins that are subsequently developed. The basic plug-in must also 
be developed with the plug-in manager at the first time otherwise the software cannot start normally.

The database-related plug-ins consists of a first-level database management plug-in and a series of secondlevel plug-ins that specifically implement database functions.

There are three levels of model-related plug-ins: level one is a model management plug-in; level two is a plugin that directly processes a specific format file and an external model interface plug-in; level three is a thirdparty software external interface plug-in that is used to process and parse external interfaces.

\section{Software engineering}

In the choice of system development tools, Python and PyQt are suitable for the development of graphical modeling and simulation software for power systems. $\mathrm{C}++$ and Qt can also be used to develop simulation systems. Considering that $\mathrm{C}++$ has a higher execution efficiency and a more convenient software release process than Python, $\mathrm{C}++$ and Qt are used for program development, which have a powerful graphical user interface and easy to implement cross-platform. Finally, a combination of $\mathrm{C}++$ and $\mathrm{Qt}$ is selected for this software system Development.

In order to comply with the orientation of the integrated energy system, the software system must have a graphical user interface (GUI) system with a unified style and complete functions. This article uses the powerful QT programming framework to implement GUI programming. In addition to the basic GUI elements such as windows, dialog boxes, and various graphical controls, the user interface is more important to the integrated design of graphics and models that can support subsystems in various fields of the integrated energy system.

Since the software system uses a plug-in-based software framework, the core plug-in is initially set to implement the overall function of the GUI, and the corresponding interface is reserved according to the set rules, so that the plug-in can be expanded later to realize the specific functions of each.

\section{Example project analysis}

This article takes an industrial base located in Nanjing as an example project. The total construction area of the industrial base is about 688.8088 square meters, including 588.5889 square meters above ground and 102.99 million square meters underground. There are many types of buildings in the industrial base, including: production scheduling centre, product testing area, production and processing debugging area, logistics support area, dormitory area, scientific research and development area and garage. There is an integrated energy demand for power-heat-cold, and the peak-tovalley difference of power grid load is large. In order to efficiently meet the energy demand of the industrial base, the base is equipped with photovoltaic, electric boilers, heat storage tanks, electric refrigeration equipment, and ice storage equipment. The partial wiring diagram of the ICES system is shown in Figure 4.

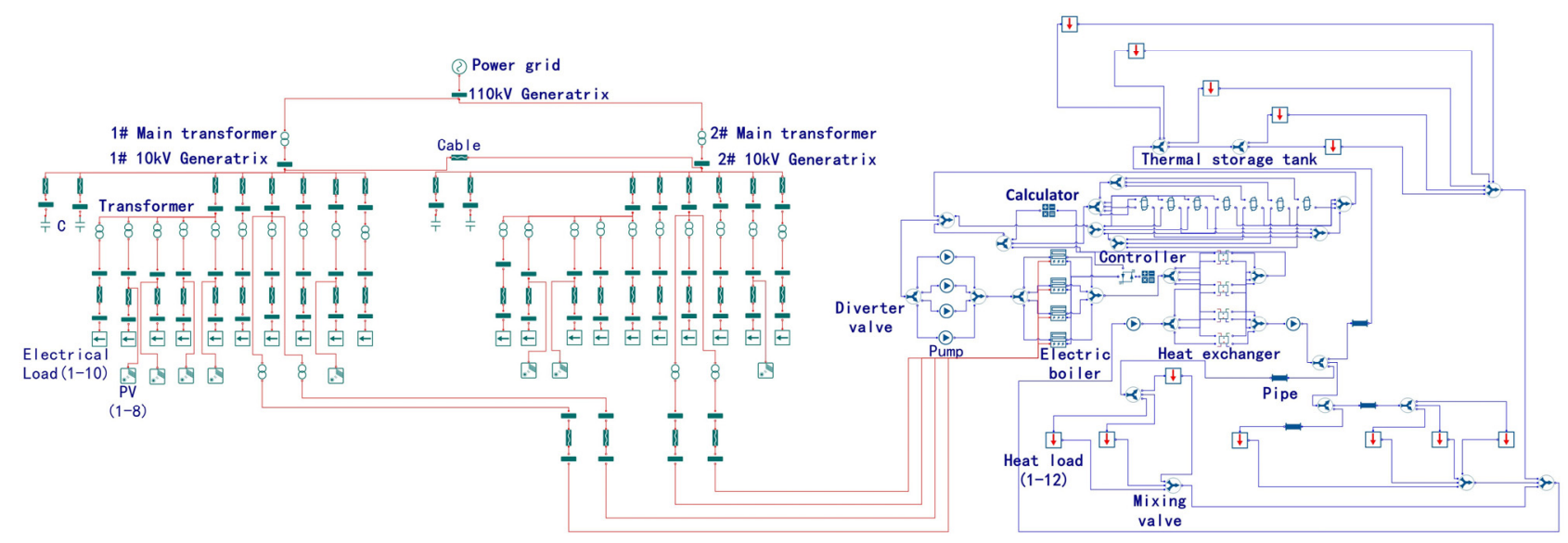

Fig.4. The system diagram of example project.

Some parameters of the equipment for the example are shown in Table 1.

Table 1. Parameters of equipment.

\begin{tabular}{|c|c|c|}
\hline Equipment name & Rated Capacity & Unit \\
\hline Photovoltaic & 2.3 & MW \\
\hline Electric boiler & $4 * 2.655$ & MW \\
\hline
\end{tabular}

\begin{tabular}{|c|c|c|}
\hline $\begin{array}{c}\text { Electric } \\
\text { refrigerator }\end{array}$ & $8 * 1100$ & $\mathrm{RT}$ \\
\hline Ice storage & 45000 & $\mathrm{RTh}$ \\
\hline Thermal storage & $7 * 400$ & $\mathrm{~m}^{3}$ \\
\hline Heat exchanger & $8 * 1900$ & $\mathrm{RT}$ \\
\hline Transformer & $2 * 16$ & $\mathrm{MW}$ \\
\hline
\end{tabular}


This sample project involves simulation calculations in the fields of power and heat-cold. You can build models on the heat-cold modeling layer and the power modeling layer. Before designing the layers, first determine the coupling elements in the sample project. The coupling elements in this example project are: electric boiler + heat storage tank and electric refrigeration + ice storage equipment. The user sets the parameter coupling component parameters, so that the entire modeling system conforms to the principle of energy cascade utilization, so that the models of different layers are linked together. In this example, the user builds a model of the project in the example on the power layer and the heat-cold layer, and encapsulates it into a power and heat-cold FMU. Finally, the integrated simulation model of power-cold-heat-gas is displayed on the same interface, and the simulation calculation is completed based on FMU technology in the background. The simulation results of the sample project are shown in Figure 5.

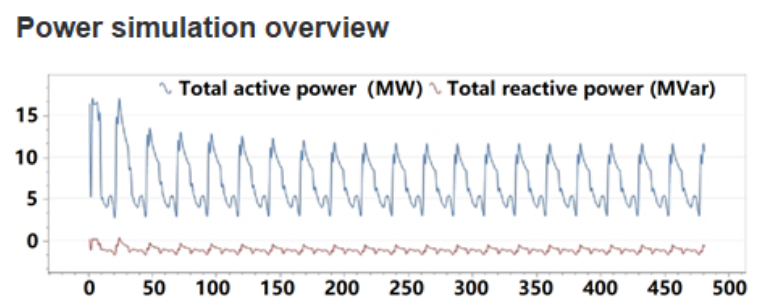

\begin{tabular}{|l|l|l|l|l|l|}
\hline Type of power value & Minimum & Maximum & Average & Quantity & Total \\
\hline Total active power & 3.35 & 16.97 & 7.11 & 481 & 3418.00 \\
\hline Total reactive power & -1.68 & 0.30 & -1.13 & 481 & -541.71 \\
\hline
\end{tabular}

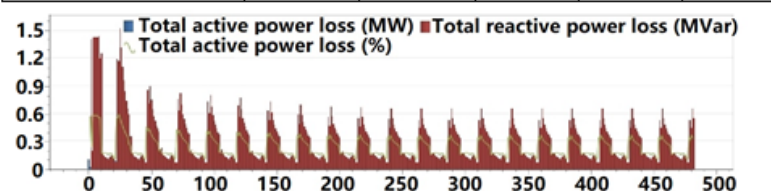

\begin{tabular}{|l|l|l|l|l|l|}
\hline Type of loss value & Minimum & Maximum & Average & Quantity & Total \\
\hline Total active power & 0.004 & 0.100 & 0.019 & 481 & 9.340 \\
\hline Total reactive power & 0.058 & 1.513 & 0.299 & 481 & 143.636 \\
\hline
\end{tabular}

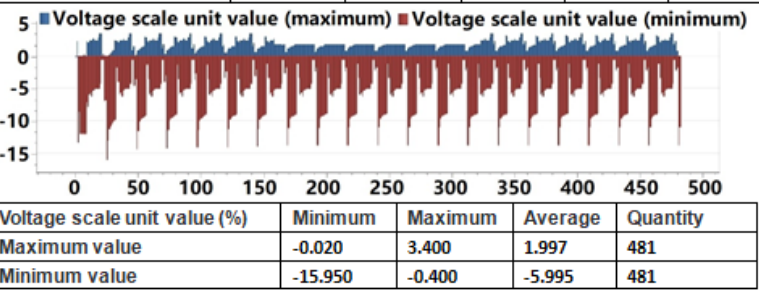

Fig.5. Simulation results.

\section{Conclusion}

The simulation system can provide a practical and feasible simulation solution for integrated energy engineering in distribution network. According to the idea of three cascades of energy utilization, the modeling of equipment in various professional fields is completed under unified software architecture, and the simulation system is finally developed. The system is built with plug-in software, which is open and extensible. The simulation system developed in this paper can be used in the simulation of distribution integrated energy system to realize the interaction between energy flow and information flow. The simulation system can effectively improve the efficiency of integrated energy system planning and design work, and provide technical support for integrated energy system engineering.

\section{Acknowledgement}

Thanks for the support of "Research on Integrated Modeling Technology of Multi-energy Complementary Integrated Energy System" (520626190046), which is a science and technology project of State Grid Shandong Electric Power Company.

\section{References}

1. Wang Weiliang, Wang Dan, Jia Hongjie, et al. Review of steady-state analysis of typical regional integrated energy system under the background of energy internet $[\mathrm{J}]$. Proceedings of the CSEE, 2016, 36(12) : 3292-3305(in Chinese).

2. ZHANG N, CAI R. Analytical solutions and typical characteristics of part-load performances of single shaft gas turbine and its cogeneration[J]. Energy Conversion Management, 2002. 43(9-12): 13231337.

3. ROSATO A, SIBILIO S, CIAMPI G. Energy, environmental and economic dynamic performance assessment of different micro-cogeneration systems in a residential application $[\mathrm{J}]$. Applied Thermal Engineering, 2013, 59(1-2): 599-617.

4. ZHUO C. Absorption heat transformer with TFEPyr as the working pair[D]. Delft: Delft University of Technology, 1998.

5. RIFKIN J. The third industrial revolution[J] . International Study Reference, 2008. 3(7): 26-27.

6. JIA Hongjie, WANG Dan, XU Xiandong, et a1. Research on some key problems related integrated energy systems $[\mathrm{J}]$. Automation of Eleelric Power systems, 2015, 4(7): 198-207.

7. GEIDL M. Integrated modeling and optimization of multi-carrier energy systems[D]. Graz: Graz University of Technology, 2007.

8. MOEINI-AGHTAIE M, ABBASPOUR A, FOTUHI-FIRUZABAD $\mathrm{M}$, et al. A decomposed solution to multiple-energy carriers optimal power flow[J]. IEEE Transactions on Power Systems, 2014, 29(2): 707-716.

9. QUELHAS A, MC-CALLEY J D. A multiperiod generalized network flow model of the u.s. integrated energy system[J]IEEE Transactions on Power Systems, 2007, 22(2): 837 - 844. 